\title{
Evidence for a biopsychosocial influence on shoulder pain: Pain catastrophizing and catechol-O-methyltransferase (COMT) diplotype predict clinical pain ratings
}

\author{
Steven Z. George ${ }^{a},{ }^{*}$, Margaret R. Wallace ${ }^{b}$, Thomas W. Wright ${ }^{c}$, Michael W. Moser ${ }^{c}$, Warren \\ H. Greenfield IIIC, Brandon K. Sack ${ }^{\mathrm{b}}$, Deborah M. Herbstman ${ }^{\mathrm{b}}$, and Roger B. Fillingim ${ }^{\mathrm{d}}$ \\ a Department of Physical Therapy, Brooks Center for Rehabilitation Studies, University of Florida, \\ Gainesville, FL, USA \\ b Department of Molecular Genetics and Microbiology, Center for Mammalian Genetics, University of \\ Florida, Gainesville, FL, USA \\ c Department of Orthopaedics and Rehabilitation, University of Florida, Gainesville, FL, USA \\ $\mathrm{d}$ Department of Community Dentistry and Behavioral Science, University of Florida, Gainesville, FL, USA
}

\begin{abstract}
The experience of pain is believed to be influenced by social, cultural, environmental, psychological, and genetic factors. Despite this assertion, few studies have included clinically relevant pain phenotypes when investigating interactions among these variables. This study investigated whether psychological variables specific to fear-avoidance models and catechol- $O$-methyltransferase (COMT) genotype influenced pain ratings for a cohort of patients receiving operative treatment of shoulder pain. Patients $(n=58)$ completed questionnaires and had COMT genotype determined preoperatively. Then, shoulder pain ratings were collected $3-5$ months post-operatively. This cohort consisted of 24 females and 34 males, with mean age of $50.3(\mathrm{SD}=15.0)$ and pre-operative pain rating of 4.5/10 ( $\mathrm{SD}=1.8)$. The frequency of COMT diplotypes was 34 with "high COMT activity" (LPS group) and 24 with "low COMT activity" (APS/HPS group). Preliminary analysis indicated that of all the fear-avoidance variables considered (fear of pain, kinesiophobia, pain catastrophizing, and anxiety), only pain catastrophizing was a unique contributor to clinical pain ratings. A hierarchical regression model indicated that an interaction between pain catastrophizing and COMT diplotype contributed additional variance in pre-operative pain ratings. The pain catastrophizing $\times$ COMT diplotype interaction demonstrated predictive validity as patients with high pain catastrophizing and low COMT activity (APS/HPS group) were more likely (RR $=6.8,95 \% \mathrm{CI}$ $=2.8-16.7$ ) to have post-operative pain ratings of $4.0 / 10$ or higher. Our findings suggest that an interaction between pain catastrophizing and COMT diplotype has the potential to influence pain ratings in patients seeking operative treatment of their shoulder pain.
\end{abstract}

\section{Keywords}

Biopsychosocial model; Catechol- $O$-methyltransferase (COMT); Pain catastrophizing; Diplotypes; Single nucleotide polymorphism; Chronic pain; Prognosis; Shoulder surgery

*Corresponding author. Address: P.O. Box 100154, Health Science Center, Gainesville, FL 32610-0154. Tel.: +1 352 273 6432; fax: +1 352273 6109. E-mail address: E-mail: szgeorge@ @ phpp.ufl.edu (S.Z. George). 


\section{Introduction}

The experience of musculoskeletal pain varies considerably among individuals. Social, cultural, environmental, psychological, and genetic factors are all believed to contribute to this variability. Shoulder pain represents a commonly occurring musculoskeletal disorder, with 1 year prevalence estimates ranging from 5\% to 47\% (van der Heijden, 1999; Kuijpers et al., 2004) and point prevalence estimates ranging from 14\% to 21\% (Urwin et al., 1998; Picavet and Schouten, 2003). Outcomes from new shoulder pain episodes vary, with 30-50\% of individuals reporting limitations in activities of daily living (Croft et al., 1996) and only 60\% of individuals reporting full recovery in 1 year (van der Windt et al., 1996). Early and accurate identification of poor outcome from shoulder pain is currently not possible because valid prognostic factors have not been reported in the literature (Kuijpers et al., 2004).

The influence of psychological factors on pain perception and the development of chronic musculoskeletal pain has been previously reported (Linton, 2000; Pincus et al., 2002).

Recently, studies of musculoskeletal pain have focused on fear-avoidance models, with data supporting the validity of these models for patients with low back (Fritz et al., 2001; Staerkle et al., 2004), knee (Granot and Ferber, 2005; Kvist et al., 2005), and cervical pain (George et al., 2001; Nederhand et al., 2004). Fear-avoidance models have ambiguous support for patients with shoulder pain. One study involving induced muscle pain offered support for the model (George et al., 2007), while other studies involving general practice patients reported weak associations of fear-avoidance specific variables and shoulder outcomes (Kuijpers et al., 2006; van der Windt et al., 2007).

Recent studies have suggested that genetic factors also influence pain perception. Candidate pain genes have been identified and one gene with a high priority score is the catechol- $O$ methyltransferase (COMT) gene, in which polymorphisms affect COMT enzyme activity (Belfer et al., 2004). COMT is an enzyme with wide-ranging biological functions and as a result could potentially be involved in a number of pathophysiological processes (Zhu, 2002). One of its functions is metabolizing catecholamines involved in pain modulation both directly and indirectly, via effects on endogenous $\mu$-opioid function. It has been suggested that polymorphisms resulting in reduced COMT enzyme production lead to chronic over-activity of the $\mu$-opioid system, decreasing its ability to modulate nociceptive input. This assertion was confirmed in healthy volunteers as $\mu$-opioid receptor binding potential and activation were predictably associated with a COMT gene single nucleotide polymorphism (SNP) at codon 158 (Zubieta et al., 2003) and experimental pain sensitivity was associated with COMT haplotypes (Diatchenko et al., 2005, 2006). In contrast, other studies have found no association with COMT genotype and pain sensitivity in healthy volunteers (Kim et al., 2004) or weak associations in a post-surgical pain model (Kim et al., 2006).

Despite the assertion that pain perception and the subsequent development of chronic pain conditions are influenced by multiple variables, few studies have included clinically relevant pain phenotypes when investigating specific interactions involving these variables. Therefore, our purpose was to investigate whether psychological variables specific to fear-avoidance models and COMT genotype influence clinical pain ratings for a cohort of patients receiving operative treatment of shoulder pain. Our a priori hypothesis was that a statistical interaction would exist, such that patients with higher levels of psychological distress and a COMT genetic predisposition to heightened pain sensitivity would have the highest clinical pain ratings. 


\section{Methods}

\subsection{Subjects}

This study was a prospective cohort of consecutive patients seeking treatment of shoulder disorders at the University of Florida's Orthopaedics Sports Medicine Institute (OSMI) in Gainesville, FL. Subjects were recruited for 15 consecutive months with the goal of recruiting a cohort of patients with discrete shoulder pain that was primarily managed with arthroscopic surgery.

The following were the inclusion criteria for this study: (a) between 18 and 85 years of age, (b) complaints of pain limited to anterior, lateral, or posterior shoulder, (c) documented or suspected rotator cuff tendinopathy (evidence from clinical examination or imaging studies) including small $(<1 \mathrm{~cm})$, medium $(1-3 \mathrm{~cm})$, and large $(3-5 \mathrm{~cm})$ tears, (d) documented or suspected adhesive capsulitis (evidence from clinical examination or imaging studies), (e) documented or suspected SLAP lesion (evidence from clinical examination or imaging studies), and (f) scheduled for arthroscopic procedure.

The following were the exclusion criteria for this study: (a) current complaints of pain lasting greater than the past 3 months involving neck, elbow, hand, low back, hip, knee, or ankle, (b) massive or complete rotator cuff tear $(>5 \mathrm{~cm}$ ), (c) documented shoulder OA or RA, (d) prior shoulder surgery within the past year or currently complaining of pain from prior shoulder surgery, (e) current shoulder fracture, tumor, or infection, (f) previously diagnosed chronic pain disorder (including, but not limited to IBS, fibromyalgia, TMD, CLBP, etc.), (g) current psychiatric management, and (h) current gastrointestinal or renal illness.

Three of the authors (T.W.W., M.W.M., and W.H.G.) evaluated patients for study eligibility during routine clinical visits to the OSMI. As appropriate, patients provided informed consent for study participation following guidelines set forth by the University of Florida's Institutional Review Board for Human Subjects. Patients had arthroscopic shoulder surgery performed by two of the authors who were board-certified orthopaedic surgeons (T.W.W. and M.W.M.). Post-operative management was under the supervision of these authors, with standard recommendations for pain management, rehabilitation, and return to activity.

The general post-operative regimen for pain included the use of inter-scalene regional anesthetics immediately and narcotic medication in decreasing quantities until patients were 6 weeks post-operative. After 6 weeks patients' pain was controlled with non-narcotic pain medication. Rehabilitation started immediately post-operatively with use of pain relieving modalities and passive range of motion of the shoulder joint. This part of rehabilitation lasted for 2-6 weeks depending on the pathology and was advanced to an active range of motion program for an additional 6 weeks. After that period, a strengthening program was initiated with release to full activities occurring at 3-4.5 months depending on the procedure performed and the patient's prior activity level. Compliance to post-operative management recommendations was not measured as part of this study.

\subsection{Measures}

Patients completed demographic information, self-report questionnaires, and genetic samples pre-operatively (range: 1-4 days before surgery date). The post-operative assessment included clinical shoulder pain ratings (range: 3-5 months following the date of surgery). All measures were collected by two of the authors (S.Z.G. and W.H.G.), or by research assistants under their direct supervision.

2.2.1. Demographic information-Sex, age, sex, self-report of race, medication status, work-status, marital status, and involved upper extremity were collected from each patient. 
2.2.2. Self-report questionnaires-We investigated several fear-avoidance specific psychological variables (fear of pain, kinesiophobia, pain catastrophizing, and anxiety) because of their relevance in other musculoskeletal conditions and their hypothesized influence on shoulder pain (Kuijpers et al., 2004). These psychological factors were assessed with previously validated self-report questionnaires commonly used in pain research. Fear of pain was assessed with the Fear of Pain Questionnaire (FPQ-III) (McNeil and Rainwater, 1998; Osman et al., 2002; Albaret et al., 2004), kinesiophobia was assessed with the shortened version of the Tampa Scale of Kinesiophobia (TSK-11) (Goubert et al., 2004; Roelofs et al., 2004; Woby et al., 2005), pain catastrophizing was assessed with the Pain Catastrophizing Scale (PCS) (Sullivan et al., 1995; Van Damme et al., 2002; D’Eon et al., 2004). Trait anxiety was assessed with the State-Trait Anxiety Index (STAI) (Spielberger et al., 1983). All questionnaires were reported as total scores for the purposes of this study.

Clinical shoulder pain ratings were assessed with the Brief Pain Inventory (BPI), which includes a numerical rating scale (NRS) for pain intensity (Cleeland and Ryan, 1994; Cleeland et al., 1996; Tan et al., 2004). Subjects used the BPI to rate their shoulder pain intensity over three conditions, the present pain intensity, the worst pain intensity over the past $24 \mathrm{~h}$, and the best pain intensity over the past $24 \mathrm{~h}$. These three ratings were summed and divided by three (arithmetic mean) because that methodology was most consistent with the purposes of this study (Jensen et al., 1996, 1999).

2.2.3. Genetic samples-Subject DNA was extracted from buccal swabs using the Gentra PureGene system (Minneapolis, MN). The focus of the genetic analysis was on the COMT gene because it is a commonly investigated pain candidate gene, with recent studies suggesting a meaningful influence on human pain perception (Zubieta et al., 2003; Diatchenko et al., $2005,2006)$. Furthermore, its role in modulating nociceptive input was theoretically complementary to the psychological factors we were studying.

Genotyping was performed by two authors (B.K.S. and D.M.H.) as follows. Two SNPs in the COMT gene were chosen, rs4633 and rs4818, because they allowed us to create the COMT haplotypes of interest. The selected SNPs were genotyped using PCR amplification of the regions containing the SNPs, followed by restriction digestion and gel electrophoresis to distinguish the alleles. The MIT Primer3 program

(frodo.wi.mit.edu/cgi-bin/primer3/primer3_www.cgi) was used to design PCR primers for the genotyping assays.

For rs4633, the PCR primers were COMTrs4633 5': 5'-TATCGGCTGGAACGAGTTCAT and COMTrs4633-3': 5'-CTTCTGCTCGCAGTAGGTGTC. Standard PCR conditions were used, with annealing temperature of $61{ }^{\circ} \mathrm{C}$. The $341 \mathrm{bp}$ PCR product was digested with restriction enzyme BsaAI (New England Biolabs), yielding two fragments of sizes 79 and 82 bp for the C allele. For rs4818, the PCR primers were COMTrs4818-5': 5'-

CAACCCTGCACAGGCAAGAT and COMTrs4818-3': 5'-

GCCCTTTTTCCAGGTCTGACA. Standard PCR conditions were used, with an annealing temperature of $61^{\circ} \mathrm{C}$. The $296 \mathrm{bp}$ PCR product was digested with BclI, yielding two fragments of sizes 116 and $145 \mathrm{bp}$ for the $\mathrm{G}$ allele. These products were resolved by electrophoresis on ethidium-bromide-stained $8 \%$ native polyacrylamide gels.

Based on previously published linkage disequilibrium and pain-related data (Diatchenko et al., 2005), haplotypes and diplotypes were inferred from the resulting genotypes at the two loci. The previously reported COMT haplotypes are LPS (low pain sensitivity), APS (average pain sensitivity), and HPS (high pain sensitivity). Hardy-Weinberg equilibrium was analyzed using standard chi-square analysis for each SNP in this group, and the resulting $p$-values were not significant $(p>0.05)$, suggesting that these loci were in equilibrium in the population. 
The genetic data were then prepared for statistical analysis by dichotomizing the subjects into those with (1) high COMT activity and predicted to have low pain sensitivity (LPS group), and (2) low COMT activity and predicted to have high pain sensitivity (APS/HPS group). This decision was made because our sample size was not conducive to analyzing all COMT haplotypes independently and previous work has indicated that high COMT activity (LPS group) was protective of the development of a clinical pain syndrome, temporomandibular pain (Diatchenko et al., 2005).

2.2.4. Data analysis-Descriptive statistics were generated for demographic, self-report, and genetic data. Differences in pre-operative clinical pain reports by pain medication status were investigated by independent $t$-test. COMT genotype frequency for our clinical sample was compared to previously reported frequencies for healthy volunteers (Diatchenko et al., 2005) by chi-square analysis. Fear of pain, kinesiophobia, pain catastrophizing, and anxiety were entered into a simultaneous regression model with pre-operative clinical pain rating as the dependent variable. The a priori criterion for consideration in the subsequent regression model was any fear-avoidance variable that uniquely contributed to pre-operative clinical pain ratings.

The primary analysis involved a multivariate, hierarchical regression model to predict preoperative clinical pain ratings. Sex and age were first entered into the model to account for any variance in pain ratings attributed to these factors. Second, the appropriate psychological and COMT diplotype were entered. Last, the interaction between the psychological variable( $\mathrm{s}$ ) and COMT diplotype was entered into the regression model. Post-hoc analysis of statistically significant interactions was investigated by ANOVA models, as appropriate.

The validity of the psychological $\times$ COMT interaction term for predicting post-operative clinical pain ratings was then investigated using a $2 \times 2$ ANOVA. Relative risk rates were calculated for identifying patients that reported greater than $4.0 / 10$ post-operative pain ratings, based on having the psychology $\times$ COMT interaction compared to not having it. A 4.0/10 pain rating cut-off was selected based on our previous clinical experiences with this particular population and data suggesting that this pain intensity rating exceeds a patient determined threshold for what constitutes "successful" treatment (Robinson et al., 2005).

Descriptive statistics and general linear model analyses were performed with SPSS for Windows, Version 13.0 (SPSS Inc., Chicago, IL). Relative risk estimates were generated with Confidence Interval Analysis 2.0.0 (Trevor Bryant, University of Southampton). All analyses were performed with a type I error rate of 0.05 .

\section{Results}

Over the 15 month recruitment period, 59 eligible patients gave informed consent for study participation. One patient was removed from the analysis due to having an extremely rare COMT genotype that was not conducive to categorization in the previously described COMT haplotypes (Diatchenko et al., 2005, 2006). Descriptive statistics for the remaining cohort ( $n$ $=58$ ) is summarized in Table 1. The distribution of pre-operative testing was 4 days for 30/58 (51.7\%), 3 days for $1 / 58(1.7 \%), 2$ days for $14 / 58$ (24.2\%), and 1 day for $13 / 58$ subjects (22.4\%). There were no statistical differences in psychological questionnaires or pre-operative pain intensity ratings based on day of testing $(p>0.05)$. There were no statistical differences $(p>$ 0.05 ) in preoperative clinical pain ratings for patients taking pain medication for their shoulder. Also, there were no statistical differences $(p>0.05)$ in COMT frequencies for this sample, in comparison to a previously reported sample (Diatchenko et al., 2005). Only pain catastrophizing made a unique contribution to pre-operative clinical pain ratings (Table 2). Therefore, pain catastrophizing was used in the subsequent regression analysis. 
The first step (age and sex) of the hierarchical regression model explained $13.5 \%$ of the variance in pre-operative clinical pain ratings. The second step (pain catastrophizing and COMT diplotype main effects) explained an additional $32.9 \%$ of the variance $(p<0.001$ for the change). Pain catastrophizing (beta $=0.53, p<0.001$ ) and COMT diplotype (beta $=0.26, p=$ 0.032 ) factors were both unique predictors at this stage. The third step (pain catastrophizing $\times$ COMT diplotype) explained an additional $4.6 \%$ variance ( $p=0.033$ for the change). In the final model, sex (beta $=-0.25, p=0.026$ ), pain catastrophizing (beta $=0.35, p=0.008$ ), and the pain catastrophizing $\times$ COMT diplotype interaction term (beta $=0.41, p=0.033$ ) were the only unique contributors to pre-operative clinical pain ratings. This regression model was repeated on only the Caucasian patients $(n=51)$, to investigate the potential of an ethnic confound for our sample. There were no substantive changes in the regression model (data not reported) when this was done. All subjects $(n=58)$ were included in the final regression model for pre-operative clinical pain ratings in Table 3.

Post-hoc analysis of the interaction was performed by a $2 \times 2$ ANOVA to report on the magnitude of differences in pre-operative pain ratings by the pain catastrophizing $\times$ COMT diplotype interaction (Fig. 1a). For the post-hoc analyses, the PCS was dichotomized into high and low scores (70th percentile) based on guidelines from a disability prevention program (Sullivan et al., 2006). Patients having high PCS scores and low COMT activity (APS/HPS group) had significantly higher pre-operative pain ratings $(n=8$, mean $=6.7, \mathrm{SD}=0.95)$ in comparison to the high PCS and high COMT activity (LPS group) pain ratings ( $n=10$, mean $=4.6, \mathrm{SD}=1.4$ ), the low PCS and low COMT activity (APS/HPS group) pain ratings ( $n=16$, mean $=4.0, \mathrm{SD}=1.4$ ), or the low PCS and high COMT activity (LPS group) pain ratings ( $n$ $=24$, mean $=3.9, \mathrm{SD}=1.7$ ). There were no other significant differences among these groups.

Forty-seven out of $58(81.0 \%)$ subjects were re-evaluated for clinical pain ratings during the follow-up period. The distribution of post-operative assessment was 3 months for 20/47 (42.5\%), 4 months for 14/47 (29.8\%), and 5 months for $14 / 58$ (27.7\%). There was no statistical difference in post-operative pain intensity ratings based on month of testing $(p>0.05)$. Subjects that completed the study did not differ $(p>0.05)$ from those lost to follow-up on age, sex, preoperative clinical pain ratings, or COMT diplotype. Average clinical pain intensity decreased from mean pre-operative rating of 4.4/10 to mean post-operative rating of 2.6/10 $(p<0.001)$. The previously described pain catastrophizing $\times$ COMT diplotype interaction was a significant predictor of post-operative pain ratings (Fig. 1b). As with the pre-operative interaction, subject with high PCS scores and low COMT activity (APS/HPS group) had significantly higher postoperative pain ratings $(n=6$, mean $=5.1, \mathrm{SD}=2.2)$, in comparison to the high PCS and high COMT activity (LPS group) pain ratings $(n=8$, mean $=2.5, \mathrm{SD}=1.9)$, the low PCS and low COMT activity (APS/HPS group) pain ratings ( $n=13$, mean $=2.2, \mathrm{SD}=1.6$ ), or the low PCS and high COMT activity (LPS group) pain ratings $(n=20$, mean $=2.1, \mathrm{SD}=2.0)$. There were no other significant differences among these groups. The relative risk of rating post-operative shoulder pain of greater than 4.0 was 6.8 (95\% 2.8-16.7) for those patients having high preoperative PCS scores and low COMT activity (APS/HPS group), in comparison to all other groups.

\section{Discussion}

Prognostic factors for shoulder pain are generally under-reported in the literature (Kuijpers et al., 2004). This current study considered the role that psychological and genetic factors play in the development of chronic post-operative shoulder pain. General psychological stress has been previously associated with shoulder pain (Badcock et al., 2002; Kuijpers et al., 2006), but few investigations to date have considered specific psychological models. We selected a fearavoidance model because of its relevance in other musculoskeletal pain conditions, and our data suggest that pain catastrophizing was a unique contributor to clinical shoulder pain ratings 
for this cohort. Pain catastrophizing is considered a maladaptive cognitive coping style comprised of magnification, rumination, and helplessness components (Sullivan et al., 2001). Pain catastrophizing's influence on pain perception and clinical outcomes has been well documented for other clinical samples (Keefe et al., 1989, 2000; Sullivan et al., 1998, 2002; Picavet et al., 2002), but not for patients with shoulder pain seeking treatment from a general practitioner (van der Windt et al., 2007). Therefore, it can be considered a somewhat novel finding that pain catastrophizing had a positive association with pain intensity in this cohort of patients seeking operative treatment of their shoulder pain. We selected the COMT gene because of its high priority candidate rating (Belfer et al., 2004) and its previous influence on pain perception for healthy subjects (Zubieta et al., 2003; Diatchenko et al., 2005, 2006). The influence of COMT genotype for clinically relevant phenotypes has been questioned in the literature (Kim et al., 2006), but our data suggested that COMT diplotype had an influence on pain ratings, at least when determined by LPS and APS/HPS groups.

Our primary hypothesis did not involve testing main effects, however. We were most interested in investigating the potential of an interaction between these pain-related psychological and genetic factors. Biopsychosocial models have been proposed for musculoskeletal pain, yet few have investigated the extent to which specific genetic and psychological factors may interact to influence pain perception. Our findings provided preliminary support for a biopsychosocial model involving catastrophizing and COMT diplotypes. The observed interaction is partially what would be expected from a combination of these factors. One would predict higher pain ratings among patients that frequently endorse cognitions consistent with pain catastrophizing (i.e. those with high PCS scores) and have a decreased ability to modulate pain through endogenous pain inhibitory systems (i.e. those with low COMT activity).

Indeed, patients with high PCS scores and low COMT activity (APS/HPS group) had significantly higher pre- and post-operative clinical shoulder pain ratings when compared to the other three groups. Interestingly, our data did not support the presence of the other part of this interaction. That is, patients with low PCS scores and high COMT activity (LPS group) did not have lower pain ratings in comparison to the high PCS scores and high COMT activity (LPS group) or the low PCS and low COMT activity (APS/HPS group) pain ratings. Therefore, it appears that having both "protective" factors (low PCS scores and/or high COMT activity) did not favorably alter the probability of reporting lower post-operative shoulder pain ratings. We verified this finding by ensuring that COMT genotype was not associated with PCS scores, and found no difference in PCS scores $(t=0.211, p=0.834)$ in LPS vs. APS/HPS groups.

The interaction between pain catastrophizing and COMT diplotype seems to have potential for clinical relevance when the probability of having persistent pain following shoulder surgery is considered. At the post-operative assessment, patients with high baseline PCS scores and low COMT activity (APS/HPS group) were 6.8 times more likely to have pain ratings of $4.0 / 10$ or greater, in comparison to other groups. This information could potentially add to clinical decision making by improving the accuracy of prognosis for outcomes following arthroscopic shoulder surgery. This information would appear to be especially relevant if pain relief was a primary reason for the patient seeking operative treatment of his/her shoulder.

These results provide promising support for this biopsychosocial model, but there are also several limitations that need to be considered when interpreting these results (Belfer et al., 2004; Max, 2004). The primary limitation is our relatively small sample size. We had enough statistical power to address our primary outcome measure of clinical pain ratings, but the small sample size limited our regression models by not being able to investigate full models. For example, we were not able to consider additional interactions with sex or age, and could not consider individual COMT haplotypes in our analysis. Future studies attempting to replicate 
this interaction should consider sample sizes of greater than 100 , given the allele frequencies and the occurrence of persistent shoulder pain observed in this study (Belfer et al., 2004).

Another limitation of this study was that this was an ethnically diverse sample, which is not ideal for genetic association studies (Max, 2004). The regression analysis was similar whether we included only Caucasian patients or the entire sample, so we included all patient data in this manuscript. With a larger and more diverse sample, future studies could consider separate models for different ethnicities or include ethnicity as a variable in regression models to account for potential confounding due to ethnic differences in COMT polymorphisms and/or expression of pain (Max, 2004). We focused only on the COMT gene because of its previously mentioned associations with experimental pain sensitivity and development of facial pain. This could be considered a limitation as other high priority pain candidate genes have been identified (Belfer et al., 2004) and pain perception is likely to be influenced by variations in multiple genes.

In this study, we used haplotypes because they are directly related to COMT enzyme activity and are genetically more informative than individual SNPs. We did not measure the commonly reported COMT SNP involving val/met substitution at codon 158 (rs4680) because it has such strong linkage disequilibrium with the other SNPs that its genotype can be inferred extremely accurately. Since recent work suggests differing influence on experimental pain sensitivity for the COMT val/met substitution at codon 158 and the diplotypes included in this study (Diatchenko et al., 2006), future study will include this SNP to detect any rare recombinant haplotypes for independent analysis of the val/met SNP.

Another limitation is that although patients followed a standard post-operative management plan, we did not systematically track the post-operative course for each patient. Therefore, there could have been variation with regard to compliance of pain management and rehabilitation following arthroscopic surgery. These untracked factors could have potentially contributed to the prediction of clinical pain ratings in this study. Finally, the direct influence COMT genotype has on metabolic processes has been the topic of several other studies (Zubieta et al., 2003; Nackley et al., 2006a, b). We did not directly measure COMT enzyme activity in these subjects, which could be considered a limitation associated with this current study.

This study is one of the first we are aware of that investigated a specific psychological and genetic interaction for its influence on operative shoulder pain. Since replication of gene association studies is rare (Max, 2004), future study is necessary to validate this interaction in a larger cohort of patients. Future study in larger sample sizes should consider a wider range of pain candidate genes, beyond COMT. Methodology in future studies should include standard pain stimuli to determine if this model is applicable to experimental pain sensitivity, as well as clinical pain reports. This predictive model could also be investigated in other pain conditions because its factors (pain catastrophizing and COMT genotype) are likely to have a broad influence on pain perception that would not be specific to the anatomical region of the shoulder. It is a viable hypothesis that pain catastrophizing and COMT genotype may influence pain perception in other musculoskeletal pain conditions receiving operative care. Another viable hypothesis is that other psychological factors could interact with different pain candidate genes to influence pain perception in musculoskeletal pain conditions.

In summary, multiple factors are hypothesized to influence pain perception, but few studies have considered specific models in clinically relevant phenotypes. Our results suggest that high pain catastrophizing and a low COMT activity were associated with higher pre-operative pain ratings, and an increased chance of experiencing persistent pain following arthroscopic shoulder surgery. Currently, psychological factors, like pain catastrophizing, are utilized for risk screening in some settings (Sullivan et al., 2006). With the human genome completed, clinical settings will soon have routine access to patient genotypes associated with pain 
perception. If predictive models involving psychological and genetic factors are validated, screening for development of chronic pain may be enhanced, resulting in early application of preventative treatment strategies.

\section{Acknowledgements}

This study was funded by the University of Florida, Research Opportunity Incentive Fund, \#56577 (S.Z.G.) and by NS41670 (R.B.F.). MaryBeth Horodyski for her organizational assistance, Kevin Robinson for his assistance with clinical recruitment, Jessica Neff for her assistance in data collection and verification and Michelle N. Burch for molecular assistance are acknowledged.

\section{References}

Albaret MC, MunozSastre MT, Cottencin A, Mullet E. The fear of pain questionnaire: factor structure in samples of young, middle-aged and elderly European people. Eur J Pain 2004;8:273-81. [PubMed: 15109978]

Badcock LJ, Lewis M, Hay EM, McCarney R, Croft PR. Chronic shoulder pain in the community: a syndrome of disability or distress? Ann Rheum Dis 2002;61:128-31. [PubMed: 11796398]

Belfer I, Wu T, Kingman A, Krishnaraju RK, Goldman D, Max MB. Candidate gene studies of human pain mechanisms: methods for optimizing choice of polymorphisms and sample size. Anesthesiology 2004;100:1562-72. [PubMed: 15166579]

Cleeland CS, Nakamura Y, Mendoza TR, Edwards KR, Douglas J, Serlin RC. Dimensions of the impact of cancer pain in a four country sample: new information from multidimensional scaling. Pain 1996;67:267-73. [PubMed: 8951920]

Cleeland CS, Ryan KM. Pain assessment: global use of the Brief Pain Inventory. Ann Acad Med Singapore 1994;23:129-38. [PubMed: 8080219]

Croft P, Pope D, Silman A. The clinical course of shoulder pain: prospective cohort study in primary care. Primary care rheumatology society shoulder study group. BMJ 1996;313:601-2. [PubMed: 8806252]

D'Eon JL, Harris CA, Ellis JA. Testing factorial validity and gender invariance of the pain catastrophizing scale. J Behav Med 2004;27:361-72. [PubMed: 15559733]

Diatchenko L, Nackley AG, Slade GD, Bhalang K, Belfer I, Max MB, et al. Catechol- $O$-methyltransferase gene polymorphisms are associated with multiple pain-evoking stimuli. Pain 2006;125:216-24. [PubMed: 16837133]

Diatchenko L, Slade GD, Nackley AG, Bhalang K, Sigurdsson A, Belfer I, et al. Genetic basis for individual variations in pain perception and the development of a chronic pain condition. Hum Mol Genet 2005;14:135-43. [PubMed: 15537663]

Fritz JM, George SZ, Delitto A. The role of fear avoidance beliefs in acute low back pain: relationships with current and future disability and work status. Pain 2001;94:7-15. [PubMed: 11576740]

George SZ, Dover GC, Fillingim RB. Fear of pain influences outcomes after exercise-induced delayed onset muscle soreness at the shoulder. Clin J Pain 2007;23:76-84. [PubMed: 17277648]

George SZ, Fritz JM, Erhard RE. A comparison of fear-avoidance beliefs in patients with lumbar spine pain and cervical spine pain. Spine 2001;26:2139-45. [PubMed: 11698893]

Goubert L, Crombez G, Van Damme S, Vlaeyen JW, Bijttebier P, Roelofs J. Confirmatory factor analysis of the tampa scale for Kinesiophobia: invariant two-factor model across low back pain patients and fibromyalgia patients. Clin J Pain 2004;20:103-10. [PubMed: 14770050]

Granot M, Ferber SG. The roles of pain catastrophizing and anxiety in the prediction of postoperative pain intensity: a prospective study. Clin J Pain 2005;21:439-45. [PubMed: 16093750]

Jensen MP, Turner JA, Romano JM, Fisher LD. Comparative reliability and validity of chronic pain intensity measures. Pain 1999;83:157-62. [PubMed: 10534586]

Jensen MP, Turner LR, Turner JA, Romano JM. The use of multiple-item scales for pain intensity measurement in chronic pain patients. Pain 1996;67:35-40. [PubMed: 8895229]

Keefe FJ, Brown GK, Wallston KA, Caldwell DS. Coping with rheumatoid arthritis pain: catastrophizing as a maladaptive strategy. Pain 1989;37:51-6. [PubMed: 2726278] 
Keefe FJ, Lefebvre JC, Egert JR, Affleck G, Sullivan MJ, Caldwell DS. The relationship of gender to pain, pain behavior, and disability in osteoarthritis patients: the role of catastrophizing. Pain 2000;87:325-34. [PubMed: 10963912]

Kim H, Lee H, Rowan J, Brahim J, Dionne RA. Genetic polymorphisms in monoamine neurotransmitter systems show only weak association with acute post-surgical pain in humans. Mol Pain 2006;2:24. [PubMed: 16848906]

Kim H, Neubert JK, San Miguel A, Xu K, Krishnaraju RK, Iadarola MJ, et al. Genetic influence on variability in human acute experimental pain sensitivity associated with gender, ethnicity and psychological temperament. Pain 2004;109:488-96. [PubMed: 15157710]

Kuijpers T, van der Windt DA, Boeke AJ, Twisk JW, Vergouwe Y, Bouter LM, et al. Clinical prediction rules for the prognosis of shoulder pain in general practice. Pain 2006;120:276-85. [PubMed: 16426760]

Kuijpers T, van der Windt DA, van der Heijden GJ, Bouter LM. Systematic review of prognostic cohort studies on shoulder disorders. Pain 2004;109:420-31. [PubMed: 15157703]

Kvist J, Ek A, Sporrstedt K, Good L. Fear of re-injury: a hindrance for returning to sports after anterior cruciate ligament reconstruction. Knee Surg Sports Traumatol Arthrosc 2005;13:393-7. [PubMed: 15703963]

Linton SJ. A review of psychological risk factors in back and neck pain. Spine 2000;25:1148-56. [PubMed: 10788861]

Max MB. Assessing pain candidate gene studies. Pain 2004;109:1-3. [PubMed: 15082119]

McNeil DW, Rainwater AJ. Development of the fear of pain questionnaire - iii. J Behav Med 1998;21:389-410. [PubMed: 9789168]

Nackley AG, Shabalina SA, Tchivileva IE, Satterfield K, Korchynskyi O, Makarov SS, et al. Human catechol- $O$-methyltransferase haplotypes modulate protein expression by altering mRNA secondary structure. Science 2006a;314:1930-3. [PubMed: 17185601]

Nackley AG, Tan KS, Fecho K, Flood P, Diatchenko L, Maixner W. Catechol- $O$-methyltransferase inhibition increases pain sensitivity through activation of both beta(2)- and beta(3)-adrenergic receptors. Pain. 2006

Nederhand MJ, Ijzerman MJ, Hermens HJ, Turk DC, Zilvold G. Predictive value of fear avoidance in developing chronic neck pain disability: consequences for clinical decision making. Arch Phys Med Rehabil 2004;85:496-501. [PubMed: 15031840]

Osman A, Breitenstein JL, Barrios FX, Gutierrez PM, Kopper BA. The fear of pain questionnaire - iii: further reliability and validity with nonclinical samples. J Behav Med 2002;25:155-73. [PubMed: 11977436]

Picavet HS, Schouten JS. Musculoskeletal pain in the Netherlands: prevalences, consequences and risk groups, the DMC(3)-study. Pain 2003;102:167-78. [PubMed: 12620608]

Picavet HS, Vlaeyen JW, Schouten JS. Pain catastrophizing and kinesiophobia: predictors of chronic low back pain. Am J Epidemiol 2002;156:1028-34. [PubMed: 12446259]

Pincus T, Burton AK, Vogel S, Field AP. A systematic review of psychological factors as predictors of chronicity/disability in prospective cohorts of low back pain. Spine 2002;27:E109-20. [PubMed: 11880847]

Robinson ME, Brown JL, George SZ, Edwards PS, Atchison JW, Hirsh AT, et al. Multidimensional success criteria and expectations for treatment of chronic pain: the patient perspective. Pain Med 2005;6:336-45. [PubMed: 16266354]

Roelofs J, Goubert L, Peters ML, Vlaeyen JW, Crombez G. The Tampa Scale for Kinesiophobia: further examination of psychometric properties in patients with chronic low back pain and fibromyalgia. Eur J Pain 2004;8:495-502. [PubMed: 15324781]

Spielberger CD, Gorsuch RL, Lushene RE, Vagg PR, Jacobs GA. Manual for the state and trait anxiety inventory (form Y). 1983

Staerkle R, Mannion AF, Elfering A, Junge A, Semmer NK, Jacobshagen N, et al. Longitudinal validation of the fear-avoidance beliefs questionnaire (FABQ) in a Swiss-German sample of low back pain patients. Eur Spine J 2004;13:332-40. [PubMed: 14714246] 
Sullivan MJ, Adams H, Rhodenizer T, Stanish WD. A psychosocial risk factor-targeted intervention for the prevention of chronic pain and disability following whiplash injury. Phys Ther 2006;86:8-18. [PubMed: 16386058]

Sullivan MJ, Stanish W, Sullivan ME, Tripp D. Differential predictors of pain and disability in patients with whiplash injuries. Pain Res Manag 2002;7:68-74. [PubMed: 12185370]

Sullivan MJ, Stanish W, Waite H, Sullivan M, Tripp DA. Catastrophizing, pain, and disability in patients with soft-tissue injuries. Pain 1998;77:253-60. [PubMed: 9808350]

Sullivan MJ, Thorn B, Haythornthwaite JA, Keefe F, Martin M, Bradley LA, et al. Theoretical perspectives on the relation between catastrophizing and pain. Clin J Pain 2001;17:52-64. [PubMed: 11289089]

Sullivan MJL, Bishop SR, Pivik J. The Pain catastrophizing scale: development and validation. Psychol Assess 1995; 7:524-32.

Tan G, Jensen MP, Thornby JI, Shanti BF. Validation of the brief pain inventory for chronic nonmalignant pain. J Pain 2004;5:133-7. [PubMed: 15042521]

Urwin M, Symmons D, Allison T, Brammah T, Busby H, Roxby M, et al. Estimating the burden of musculoskeletal disorders in the community: the comparative prevalence of symptoms at different anatomical sites, and the relation to social deprivation. Ann Rheum Dis 1998;57:649-55. [PubMed: 9924205]

Van Damme S, Crombez G, Bijttebier P, Goubert L, Van Houdenhove B. A confirmatory factor analysis of the Pain Catastrophizing Scale: invariant factor structure across clinical and non-clinical populations. Pain 2002;96:319-24. [PubMed: 11973004]

van der Heijden GJ. Shoulder disorders: a state-of-the-art review. Baillieres Clin Rheumatol 1999;13:287-309. [PubMed: 10445116]

van der Windt DA, Koes BW, Boeke AJ, Deville W, De Jong BA, Bouter LM. Shoulder disorders in general practice: prognostic indicators of outcome. Br J Gen Pract 1996;46:519-23. [PubMed: 8917870]

van der Windt DA, Kuijpers T, Jellema P, van der Heijden GJ, Bouter LM. Do psychological factors predict outcome in both low-back pain and shoulder pain? Ann Rheum Dis 2007;66:313-9. [PubMed: 16916857]

Woby SR, Roach NK, Urmston M, Watson PJ. Psychometric properties of the TSK-11: a shortened version of the Tampa Scale for Kinesiophobia. Pain 2005;117:137-44. [PubMed: 16055269]

Zhu BT. Catechol- $O$-methyltransferase (COMT)-mediated methylation metabolism of endogenous bioactive catechols and modulation by endobiotics and xenobiotics: importance in pathophysiology and pathogenesis. Curr Drug Metab 2002;3:321-49. [PubMed: 12083324]

Zubieta JK, Heitzeg MM, Smith YR, Bueller JA, Xu K, Xu Y, et al. COMT val158met genotype affects mu-opioid neurotransmitter responses to a pain stressor. Science 2003;299:1240-3. [PubMed: 12595695] 

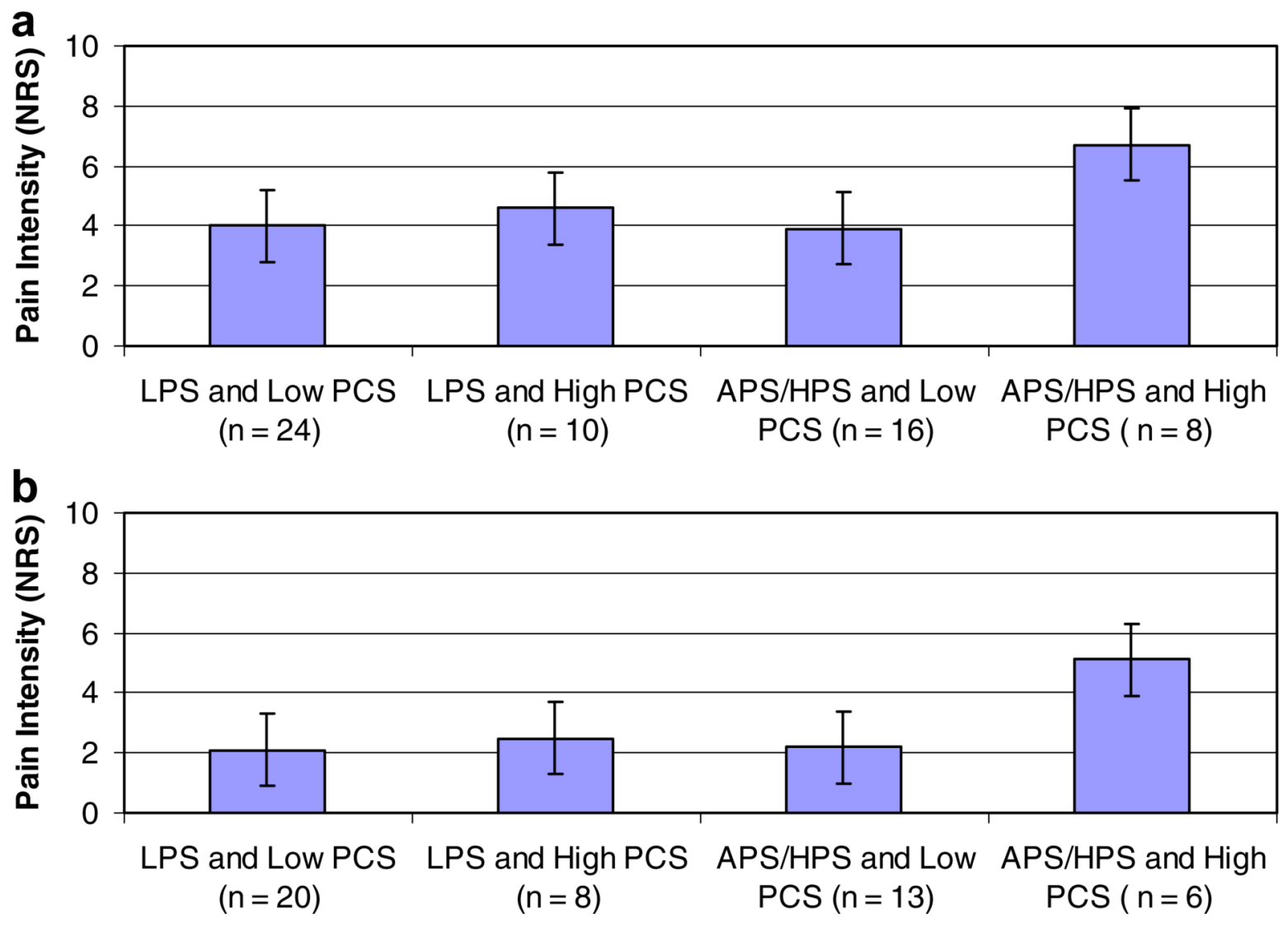

Fig. 1.

(a) Interaction of pain catastrophizing and COMT diplotype: pre-operative shoulder pain ratings. (b) Interaction of pain catastrophizing and COMT diplotype: post-operative shoulder pain ratings. Figure key: Error bars, two standard error; LPS, high COMT activity; APS/HPS, low COMT activity; low PCS, low pain catastrophizing scores from Pain Catastrophizing Scale; high PCS, high pain catastrophizing scores from Pain Catastrophizing Scale. 
Table 1

Descriptive statistics for operative shoulder pain cohort $(n=58)$

\begin{tabular}{|c|c|c|}
\hline Variable & Values & $\%$ or SD \\
\hline Sex (\# females, \%) & 24 & $41.4 \%$ \\
\hline Age & 50.3 & 15.0 \\
\hline \multicolumn{3}{|l|}{ Race } \\
\hline (\# Caucasian, \%) & 51 & 89.5 \\
\hline (\# African American, \%) & 4 & 7.0 \\
\hline (\# Not specified, \%) & 2 & 3.5 \\
\hline Taking pain medication for shoulder (\# yes, \%) & 24 & $41.4 \%$ \\
\hline Work related shoulder injury ${ }^{a}$ (\# yes, $\%$ ) & 8 & $13.8 \%$ \\
\hline Marital status $^{a}$ (\# married, \%) & 31 & $63.3 \%$ \\
\hline Involved upper extremity (\# right, \%) & 37 & $63.8 \%$ \\
\hline Fear of pain questionnaire & 71.0 & 21.4 \\
\hline Tampa scale of kinesiophobia ${ }^{b}$ & 25.1 & 6.4 \\
\hline Pain catastrophizing scale & 13.6 & 9.6 \\
\hline State-Trait Anxiety Inventory & 36.8 & 11.7 \\
\hline Average pre-operative pain rating ( $0-10 \mathrm{NRS})$ & 4.5 & 1.8 \\
\hline Low COMT activity (LPS group) & 34 & $58.6 \%$ \\
\hline (\#LPS/LPS, \%) & 11 & $19.0 \%$ \\
\hline (\#LPS/APS, \%) & 19 & $32.8 \%$ \\
\hline (\#HPS/LPS, \%) & 4 & $6.8 \%$ \\
\hline High COMT activity (APS/HPS group) & 24 & $41.4 \%$ \\
\hline (\#APS, \%) & 19 & $32.8 \%$ \\
\hline (\#HPS/APS, \%) & 5 & $8.6 \%$ \\
\hline Completed post-operative follow-up (\#, \%) & 47 & $81.0 \%$ \\
\hline Average post-operative pain rating $(0-10 \mathrm{NRS})$ & 2.6 & 2.1 \\
\hline
\end{tabular}

All values are means and standard deviations, unless otherwise indicated.

${ }^{a}$ Nine subjects did not respond to this category.

${ }^{b}$ Eleven-item version of the TSK was used in this study. 
Table 2

Fear-avoidance specific variables, influence on shoulder pain

\begin{tabular}{lccc}
\hline Overall model & & & \\
\hline R-Square $=0.276$ & & & \\
Adjusted R-Square $=0.221$ & & & \\
$F_{4,53}=5.05$ & & & $p$-value \\
$p=0.002$ & $B$ & Std. beta & 0.584 \\
Variables & 0.01 & 0.08 & 0.329 \\
Fear of pain & -0.04 & -0.15 & $\mathbf{0 . 0 0 1}$ \\
Kinesiophobia & $\mathbf{0 . 1 0}$ & $\mathbf{0 . 5 3}$ & 0.646 \\
Pain catastrophizing & 0.01 & 0.07 & \\
Anxiety & & & \\
\hline
\end{tabular}

Bold font indicates variable was selected for use in subsequent regression model. 
Table 3

Evidence for biopsychosocial influence on pre-operative clinical pain ratings

Final model

R-Square $=0.510$

Adjusted R-Square $=0.462$

$F_{5,51}=10.62$

$p<0.001$

Variables

B

Age

Sex

Pain catastrophizing

COMT diplotype

Pain catastrophizing $\times$ COMT diplotype

Bold font indicates variable was statistically significant predictor in final model.

Individual main effects of pain catastrophizing and COMT diplotype were not considered because of significant interaction term.

Sex was coded $($ males $=1$, females $=0)$.

COMT diplotype was coded (APS/HPS $=1$, LPS $=0$ ). 